\title{
Non-Hermitian Quantum Systems and Time-Optimal Quantum Evolution*
}

\author{
Alexander I. NESTEROV \\ Departamento de Física, CUCEI, Universidad de Guadalajara, \\ Av. Revolución 1500, Guadalajara, CP 44420, Jalisco, México \\ E-mail: nesterov@cencar.udg.mx
}

Received November 17, 2008, in final form June 23, 2009; Published online July 07, 2009 doi:10.3842/SIGMA.2009.069

\begin{abstract}
Recently, Bender et al. have considered the quantum brachistochrone problem for the non-Hermitian $\mathcal{P} \mathcal{T}$-symmetric quantum system and have shown that the optimal time evolution required to transform a given initial state $\left|\psi_{i}\right\rangle$ into a specific final state $\left|\psi_{f}\right\rangle$ can be made arbitrarily small. Additionally, it has been shown that finding the shortest possible time requires only the solution of the two-dimensional problem for the quantum system governed by the effective Hamiltonian acting in the subspace spanned by $\left|\psi_{i}\right\rangle$ and $\left|\psi_{f}\right\rangle$. In this paper, we study a similar problem for the generic non-Hermitian Hamiltonian, focusing our attention on the geometric aspects of the problem.
\end{abstract}

Key words: non-Hermitian quantum systems; quantum brachistochrone problem

2000 Mathematics Subject Classification: 81S10; 81V99; 53Z05

\section{Introduction}

In view of recent results on optimal quantum evolution and its possible relation to quantum computation and quantum information processing, there has been increasing interest in the quantum brachistochrone problem. The problem consists of finding the optimal time evolution $\tau$ to evolve a given initial state $\left|\psi_{i}\right\rangle$ into a certain final state $\left|\psi_{f}\right\rangle$ under a given set of constraints [1, $2,3,4,5]$. It is known that for the Hermitian Hamiltonian, $\tau$ has a nonzero lower bound. However, Bender et al. [2] have recently shown that for non-Hermitian $\mathcal{P} \mathcal{T}$-symmetric quantum systems, the answer is quite different and that the time evolution $\tau$ can be made arbitrarily small, despite the fact that the eigenvalue constraint is held fixed and identical to that for the corresponding Hermitian system. The mechanism described in [2] resembles the wormhole effect in general relativity and has generated discussion in the literature $[6,7,8,9,10,11,12,13,14]$.

Non-Hermitian Hamiltonians emerge in physics in different ways. For instance, the application for description of dissipative systems is well known from the classical works by Weisskopf and Wigner on metastable states $[15,16,17]$. It was demonstrated that the evolution of the quantum system, being initially in the metastable state $\psi(0)$, can be described by the effective non-Hermitian Hamiltonian $H_{\text {eff }}$ as follows: $\psi(0) \rightarrow \psi(t)=e^{-i H_{\text {eff }} t} \psi(0)+$ decay products. Recently, it has been shown how the non-Hermitian Hamiltonian appears in the framework of the quantum jump approach to open systems $[18,19]$. Other examples include complex refractive indices in optics, complex potentials describing the scattering of electrons, atom diffraction by light, line widths of unstable lasers, etc. For the following, it is essential that non-Hermitian physics differs drastically from the conventional physics in the presence of the so-called excep-

${ }^{\star}$ This paper is a contribution to the Proceedings of the VIIth Workshop "Quantum Physics with NonHermitian Operators" (June 29 - July 11, 2008, Benasque, Spain). The full collection is available at http://www.emis.de/journals/SIGMA/PHHQP2008.html 
tional points, where the eigenvalues and eigenvectors coalesce, even if the non-Hermiticity is regarded as a perturbation [20].

In the Hermitian quantum mechanics, the optimal time evolution problem implies finding the transformation $\left|\psi_{i}\right\rangle \rightarrow e^{-i H t}\left|\psi_{i}\right\rangle$ that provides the shortest time $t=\tau$ under a given set of constraints [1]. The generic non-Hermitian quantum brachistochrone problem poses the same question, with the exception that now the evolution of the system is described by the nonHermitian Hamiltonian, which is not necessarily $\mathcal{P} \mathcal{T}$-symmetric $[2,6,12]$.

Recently, this problem has been studied by Assis and Fring for a dissipative quantum system governed by a symmetric non-Hermitian Hamiltonian [6]. It has been shown that the passage time required to transform a given initial state to the orthogonal final state can be arbitrarily small. The obtained effect, being similar to the one discovered by Bender et al. [2], is related to the non-Hermitian nature of the system rather than to its $\mathcal{P} \mathcal{T}$-symmetry.

In this paper, we address the non-Hermitian quantum brachistochrone problem, considering the generic non-Hermitian Hamiltonian, and focus our attention on the geometric aspects of the problem. In Section 2 we first briefly summarize the main properties of non-Hermitian quantum systems. We discuss the non-Hermitian quantum brachistochrone problem and the associated two-dimensional effective Hamiltonian, acting in the two-dimensional space spanned by the initial and final states. In Section 3 we discuss the quantum brachistochrone in the vicinity of the exceptional point. In Section 4 we consider the non-Hermitian quantum brachistochrone problem in the context of the Fubini-Study metric on the complex Bloch sphere. In Section 5 the results and open problems are discussed.

\section{Non-Hermitian quantum brachistochrone problem}

In the quantum brachistochrone problem, finding the shortest possible time requires only the solution of the two-dimensional problem, namely, finding the optimal time evolution for the quantum system governed by the effective Hamiltonian acting in the subspace spanned by $\left|\psi_{i}\right\rangle$ and $\left|\psi_{f}\right\rangle[2,3,4]$.

Before proceeding further, we outline some background information on non-Hermitian quantum systems. Let an adjoint pair $\{|\psi(t)\rangle,|\tilde{\psi}(t)\rangle\}$ be a solution to the Schrödinger equation and its adjoint equation

$$
i \frac{\partial}{\partial t}|\psi(t)\rangle=H|\psi(t)\rangle, \quad i \frac{\partial}{\partial t}|\tilde{\psi}(t)\rangle=H^{\dagger}|\tilde{\psi}(t)\rangle,
$$

which can be recast in the form

$$
i \frac{\partial}{\partial t}|\psi(t)\rangle=H|\psi(t)\rangle, \quad-i \frac{\partial}{\partial t}\langle\tilde{\psi}(t)|=\langle\tilde{\psi}(t)| H .
$$

For $\lambda_{k}$ being the eigenvalues of $H$, we denote by $\left|\psi_{k}\right\rangle$ and $\left\langle\tilde{\psi}_{k}\right|$ the corresponding right and left eigenvectors: $H\left|\psi_{k}\right\rangle=\lambda_{k}\left|\psi_{k}\right\rangle,\left\langle\tilde{\psi}_{k}\right| H=\lambda_{k}\left\langle\tilde{\psi}_{k}\right|$. The systems of both left and right eigenvectors form bi-orthogonal basis [21]

$$
\sum_{k} \frac{\left|\psi_{k}\right\rangle\left\langle\tilde{\psi}_{k}\right|}{\left\langle\tilde{\psi}_{k} \mid \psi_{k}\right\rangle}=1, \quad\left\langle\tilde{\psi}_{k} \mid \psi_{k^{\prime}}\right\rangle=0, \quad k \neq k^{\prime} .
$$

Let the set $\left\{\left|\psi_{i}\right\rangle,\left|\psi_{0}\right\rangle,\left\langle\tilde{\psi}_{i}\right|,\left\langle\tilde{\psi}_{0}\right|\right\}$ form the bi-orthonormal basis of the two-dimensional subspace spanned by the initial state $\left|\psi_{i}\right\rangle$ and the final state $\left|\psi_{f}\right\rangle$ :

$$
\left\langle\tilde{\psi}_{0} \mid \psi_{0}\right\rangle=\left\langle\tilde{\psi}_{i} \mid \psi_{i}\right\rangle=1, \quad\left\langle\tilde{\psi}_{0} \mid \psi_{i}\right\rangle=\left\langle\tilde{\psi}_{i} \mid \psi_{0}\right\rangle=0
$$


Using this basis, we can write the final state $\left|\psi_{f}\right\rangle$ as

$$
\left|\psi_{f}\right\rangle=\cos \frac{\alpha}{2}\left|\psi_{i}\right\rangle+e^{i \beta} \sin \frac{\alpha}{2}\left|\psi_{0}\right\rangle,
$$

where $\alpha, \beta$ are complex angles, and similarly,

$$
\left\langle\tilde{\psi}_{f}\right|=\cos \frac{\tilde{\alpha}}{2}\left\langle\tilde{\psi}_{i}\right|+e^{-i \tilde{\beta}} \sin \frac{\tilde{\alpha}}{2}\left\langle\tilde{\psi}_{0}\right| .
$$

Let $|\psi(t)\rangle=u_{1}(t)\left|\psi_{i}\right\rangle+u_{2}(t)\left|\psi_{0}\right\rangle$ and $\langle\tilde{\psi}(t)|=\tilde{u}_{1}\left\langle\tilde{\psi}_{i}\right|+\tilde{u}_{2}\left\langle\tilde{\psi}_{0}\right|$ be solutions to the Schrödinger equation and its adjoint equation (2.1), respectively. Then one can see that the vectors $|u(t)\rangle=$ $\left(\begin{array}{l}u_{1}(t) \\ u_{2}(t)\end{array}\right)$ and $\langle\tilde{u}(t)|=\left(\tilde{u}_{1}(t), \tilde{u}_{2}(t)\right)$ satisfy

$$
i \frac{\partial}{\partial t}|u(t)\rangle=H_{\mathrm{eff}}|u(t)\rangle, \quad-i \frac{\partial}{\partial t}\langle\tilde{u}(t)|=\langle\tilde{u}(t)| H_{\mathrm{eff}},
$$

where the effective two-dimensional Hamiltonian $H_{\text {eff }}$ reads:

$$
H_{\mathrm{eff}}=\frac{1}{2}\left(\begin{array}{cc}
\lambda_{0}+Z & X-i Y \\
X+i Y & \lambda_{0}-Z
\end{array}\right)
$$

Here, we set

$$
\begin{aligned}
& \lambda_{0}=\left\langle\tilde{\psi}_{i}|H| \psi_{i}\right\rangle+\left\langle\tilde{\psi}_{0}|H| \psi_{0}\right\rangle, \quad X=\left\langle\tilde{\psi}_{i}|H| \psi_{0}\right\rangle+\left\langle\tilde{\psi}_{0}|H| \psi_{i}\right\rangle, \\
& Y=i\left(\left\langle\tilde{\psi}_{i}|H| \psi_{0}\right\rangle-\left\langle\tilde{\psi}_{0}|H| \psi_{i}\right\rangle\right), \quad Z=\left\langle\tilde{\psi}_{i}|H| \psi_{i}\right\rangle-\left\langle\tilde{\psi}_{0}|H| \psi_{0}\right\rangle .
\end{aligned}
$$

Further, it is convenient to express the effective Hamiltonian $H_{\text {eff }}$ in terms of the Pauli matrices:

$$
H_{\mathrm{eff}}=\frac{\lambda_{0}}{2} \mathbf{1}+\frac{1}{2} \boldsymbol{\Omega} \cdot \boldsymbol{\sigma}
$$

where 1 denotes the identity operator and $\boldsymbol{\Omega}=(X, Y, Z)$.

It is then easy to show that the complex Bloch vector defined as $\mathbf{n}(t)=\langle\tilde{u}(t)|\boldsymbol{\sigma}| u(t)\rangle$, where $\boldsymbol{\sigma}$ are the Pauli matrices, satisfies the complex Bloch equation

$$
\frac{d \mathbf{n}(t)}{d t}=\mathbf{\Omega} \times \mathbf{n}(t),
$$

which is equivalent to the Schrödinger equation (2.3) (see, e.g., [22, 23]). In the explicit form, the components of the Bloch vector are given by

$$
n_{1}=u_{1} \tilde{u}_{2}+u_{2} \tilde{u}_{1}, \quad n_{2}=i\left(u_{1} \tilde{u}_{2}-u_{2} \tilde{u}_{1}\right), \quad n_{3}=u_{1} \tilde{u}_{1}-u_{2} \tilde{u}_{2} .
$$

The vector $\mathbf{n}(t)=\left(n_{1}(t), n_{2}(t), n_{3}(t)\right)$, being a complex unit vector, traces out a trajectory on the complex 2-dimensional sphere $S_{c}^{2}$, and the latter can be considered as the quantum phase space for the non-Hermitian two-level quantum system. From equation (2.5), we find that $\mathbf{n}_{i}=(0,0,1)$ corresponds to the initial state $\left|\psi_{i}\right\rangle$.

Analysis of the eigenvalue problem $H_{\text {eff }}\left|u_{ \pm}\right\rangle=\lambda|u\rangle_{ \pm},\left\langle\tilde{u}_{ \pm}\right| H_{\text {eff }}=\lambda_{ \pm}\left\langle\tilde{u}_{ \pm}\right|$, yields $\lambda_{ \pm}=$ $\left(\lambda_{0} \pm R\right) / 2$, where $R=\sqrt{X^{2}+Y^{2}+Z^{2}}$. The right and left eigenvectors are found to be

$$
\begin{array}{ll}
\left|u_{+}\right\rangle=\left(\begin{array}{c}
\cos \frac{\theta}{2} \\
e^{i \varphi} \sin \frac{\theta}{2}
\end{array}\right), \quad\left\langle\tilde{u}_{+}\right|=\left(\cos \frac{\theta}{2}, e^{-i \varphi} \sin \frac{\theta}{2}\right), \\
\left|u_{-}\right\rangle=\left(\begin{array}{c}
-e^{-i \varphi} \sin \frac{\theta}{2} \\
\cos \frac{\theta}{2}
\end{array}\right), & \left\langle\tilde{u}_{-}\right|=\left(-e^{i \varphi} \sin \frac{\theta}{2}, \cos \frac{\theta}{2}\right),
\end{array}
$$


where

$$
\begin{array}{ll}
\cos \frac{\theta}{2}=\sqrt{\frac{R+Z}{2 R}}, & \sin \frac{\theta}{2}=\sqrt{\frac{R-Z}{2 R}}, \\
e^{i \varphi}=\frac{X+i Y}{\sqrt{R^{2}-Z^{2}}}, & e^{-i \varphi}=\frac{X-i Y}{\sqrt{R^{2}-Z^{2}}},
\end{array}
$$

and

$$
X=R \sin \theta \cos \varphi, \quad Y=R \sin \theta \sin \varphi, \quad Z=R \cos \theta
$$

$(\theta, \varphi)$ being the complex spherical coordinates.

The coalescence of eigenvalues $\lambda_{+}$and $\lambda_{-}$occurs when $X^{2}+Y^{2}+Z^{2}=0$. There are two cases. The first one, defined by $\theta=0, \varphi=0$, yields two linearly independent eigenvectors. The related degeneracy is known as the diabolic point, and we obtain

$$
\left|u_{+}\right\rangle=\left(\begin{array}{l}
1 \\
0
\end{array}\right), \quad\left\langle\tilde{u}_{+}|=(1,0), \quad| u_{-}\right\rangle=\left(\begin{array}{l}
0 \\
1
\end{array}\right), \quad\left\langle\tilde{u}_{-}\right|=(0,1) .
$$

The second case is characterized by the coalescence of eigenvalues and the merging of the eigenvectors. The degeneracy point is known as the exceptional point, and we obtain: $\left|u_{+}\right\rangle=e^{i \kappa}\left|u_{-}\right\rangle$ and $\left\langle\tilde{u}_{+}\right|=e^{-i \kappa}\left\langle\tilde{u}_{-}\right.$, where $\kappa \in \mathbb{C}$ is a complex phase.

Let us assume that the exceptional point is given by $\boldsymbol{R}_{0}=\left(X_{0}, Y_{0}, Z_{0}\right)$. Then, if $Z_{0} \neq 0$, using equations (2.6)-(2.8), we obtain

$$
\tan \frac{\theta_{0}}{2}= \pm i, \quad e^{2 i \varphi_{0}}=\frac{X_{0}+i Y_{0}}{X_{0}-i Y_{0}}
$$

thus, at the exceptional point $\Im \theta \rightarrow \pm \infty$. If $Z_{0}=0$, we obtain $X_{0}= \pm i Y_{0}$. This implies that $\theta_{0}=\pi / 2$, and $\Im \varphi \rightarrow \pm \infty$ at the exceptional point.

Taking the Hamiltonian of equation (2.4), we find the solution of the Schrödinger equation (2.3), satisfying $|\psi(0)\rangle=\left|\psi_{i}\right\rangle$, as

$$
|\psi(t)\rangle=C_{1}(t) e^{-i \lambda_{0} t / 2}\left|\psi_{i}\right\rangle+C_{2}(t) e^{-i \lambda_{0} t / 2}\left|\psi_{0}\right\rangle,
$$

where

$$
C_{1}(t)=\cos \frac{\Omega t}{2}-i \cos \theta \sin \frac{\Omega t}{2}, \quad C_{2}(t)=-i e^{i \varphi} \sin \theta \sin \frac{\Omega t}{2},
$$

and we denote $\Omega=R=\sqrt{X^{2}+Y^{2}+Z^{2}}$.

The solution of the adjoint Schrödinger equation with the wave function $\langle\tilde{\psi}(t)|$ written as

$$
\langle\tilde{\psi}(t)|=\tilde{C}_{1}(t) e^{i \lambda_{0} t / 2}\left\langle\tilde{\psi}_{i}\right|+\tilde{C}_{2}(t) e^{i \lambda_{0} t / 2}\left\langle\tilde{\psi}_{0}\right|
$$

is given by

$$
\tilde{C}_{1}(t)=\cos \frac{\Omega t}{2}+i \cos \theta \sin \frac{\Omega t}{2}, \quad \tilde{C}_{2}(t)=i e^{-i \varphi} \sin \theta \sin \frac{\Omega t}{2} .
$$

Applying equation (2.2), we can write $|\psi(t)\rangle$ as

$$
|\psi(t)\rangle=\left(C_{1}(t)-e^{-i \beta} \cot \frac{\alpha}{2} C_{2}(t)\right) e^{-i \lambda_{0} t / 2}\left|\psi_{i}\right\rangle+\frac{e^{-i \beta}}{\sin \frac{\alpha}{2}} C_{2}(t) e^{-i \lambda_{0} t / 2}\left|\psi_{f}\right\rangle .
$$


Hence, the initial state $\left|\psi_{i}\right\rangle$ evolves into the final state $\left|\psi_{f}\right\rangle$ in the time $t=\tau$ when

$$
C_{1}(\tau)-C_{2}(\tau) e^{-i \beta} \cot \frac{\alpha}{2}=0, \quad C_{2}(\tau)=e^{i \beta} \sin \frac{\alpha}{2} .
$$

To find the time evolution $\tau$, we first solve the equations (2.14) for $e^{i(\varphi-\beta)}$. The computation yields

$$
\begin{aligned}
& e^{i(\varphi-\beta)}=\frac{-\cos \theta \cos \frac{\alpha}{2} \pm \sqrt{\cos ^{2} \theta-\sin ^{2} \frac{\alpha}{2}}}{\sin \theta \sin \frac{\alpha}{2}}, \\
& e^{-i(\varphi-\beta)}=\frac{-\cos \theta \cos \frac{\alpha}{2} \mp \sqrt{\cos ^{2} \theta-\sin ^{2} \frac{\alpha}{2}}}{\sin \theta \sin \frac{\alpha}{2}} .
\end{aligned}
$$

It should be noted that the solution related to the lower sign can be obtained from the solution corresponding to the upper sign by changing the parameter $\alpha$ as follows: $\alpha \rightarrow \alpha+2 \pi$. This implies the change of the total sign in the final state of the quantum-mechanical system: $\left|\psi_{f}\right\rangle \rightarrow-\left|\psi_{f}\right\rangle$. Thus, without loss of generality, one can consider only one sign (upper or lower) in equations (2.15), (2.16). Further, for definiteness, we will choose the upper sign. Then, substituting the result into (2.14), we get

$$
\tan \frac{\Omega \tau}{2}=\frac{i \sin ^{2} \frac{\alpha}{2}}{\cos \frac{\alpha}{2} \sqrt{\cos ^{2} \theta-\sin ^{2} \frac{\alpha}{2}}-\cos \theta} .
$$

From here, the time evolution $\tau$ is found to be

$$
\tau=\left|\frac{2}{\Omega} \arctan \left(\frac{i \sin ^{2} \frac{\alpha}{2}}{\cos \frac{\alpha}{2} \sqrt{\cos ^{2} \theta-\sin ^{2} \frac{\alpha}{2}}-\cos \theta}\right)\right| .
$$

In addition, since $\tau$ should be a real positive function, the following restriction must be imposed:

$$
\arg \Omega=\arg \arctan \left(\frac{i \sin ^{2} \frac{\alpha}{2}}{\cos \frac{\alpha}{2} \sqrt{\cos ^{2} \theta-\sin ^{2} \frac{\alpha}{2}}-\cos \theta}\right) .
$$

Now, the generic problem is to select a final vector $\left|\psi_{f}\right\rangle$, choosing the parameters $\alpha$ and $\beta$. Next, we must find the conditions that should be imposed on the parameters $(\theta, \varphi)$ to yield the smallest time $\tau$ required to evolve the state $\left|\psi_{i}\right\rangle$ into the state $\left|\psi_{f}\right\rangle$ under a given set of constraints with the Hamiltonian

$$
H_{\mathrm{eff}}=\frac{1}{2}\left(\begin{array}{cc}
\lambda_{0} & 0 \\
0 & \lambda_{0}
\end{array}\right)+\frac{\Omega}{2}\left(\begin{array}{cc}
\cos \theta & e^{-i \varphi} \sin \theta \\
e^{i \varphi} \sin \theta & -\cos \theta
\end{array}\right) .
$$

In what follows, we assume the eigenvalue constraint to be imposed as $\left|\lambda_{+}-\lambda_{-}\right|=|\Omega|^{1}$. This implies that the argument of $\Omega$ is determined by equation (2.19).

Further study of the critical points shows that there is no solution with a finite value of $|\theta|$ yielding the minimum of the time evolution [24]. Moreover, as follows from equation (2.18), in the limit $|\theta| \rightarrow \infty(|\Im \theta| \rightarrow \infty)$, the time evolution behaves as $\tau \approx|2 / \Omega \cos \theta| \rightarrow 0$. Thus, for a quantum-mechanical system governed by a non-Hermitian Hamiltonian, the time evolution $\tau$

\footnotetext{
${ }^{1}$ In contrast to the Hermitian quantum systems, here, there are more choices for imposing the eigenvalue constraint. For instance, instead of $\left|\lambda_{+}-\lambda_{-}\right|=|\Omega|$ being held fixed, one can require $\lambda_{+}-\lambda_{-}=\Omega=$ const [24].
} 
can be taken to be arbitrarily small. In addition, since for any finite value of $|\theta|$, the minimum of $\tau$ does not exist, the non-Hermitian Hamiltonian cannot be optimized.

However, for a quantum-mechanical system governed by a Hermitian Hamiltonian, the latter can be optimized. Indeed, in the Hermitian case, $\Im \varphi=\Im \theta=0$; hence, the saddle point $\theta=\pi / 2$ becomes the point of a local minimum (see Fig. 1). It then follows from (2.20) that

$$
H_{\mathrm{eff}}=\frac{1}{2}\left(\begin{array}{cc}
\lambda_{0} & 0 \\
0 & \lambda_{0}
\end{array}\right)+\frac{\Omega}{2}\left(\begin{array}{cc}
0 & e^{-i \varphi} \\
e^{i \varphi} & 0
\end{array}\right)
$$

and from equations (2.13)-(2.16), we obtain

$$
|\psi(t)\rangle=e^{-i \lambda_{0} t / 2}\left(\cos \frac{\Omega t}{2}-\cot \frac{\alpha}{2} \sin \frac{\Omega t}{2}\right)\left|\psi_{i}\right\rangle+\frac{e^{-i \lambda_{0} t / 2}}{\sin \frac{\alpha}{2}} \sin \frac{\Omega t}{2}\left|\psi_{f}\right\rangle .
$$

This fully agrees with the results obtained by Carlini et al. and Brody and Hook [1, 4].

We now consider some illustrative examples. Of a special interest is the case when the complex Bloch vector $\boldsymbol{n}$, entering in the non-Hermitian Hamiltonian (2.4), is orthogonal to the plane spanned by $\boldsymbol{n}_{i}$ and $\boldsymbol{n}_{f}$. Without loss of generality, one can choose $\boldsymbol{n}=\boldsymbol{n}_{i} \times \boldsymbol{n}_{f} / \sin \alpha$ and set $\boldsymbol{n}_{i}=(0,0,1)$. This yields $\theta=\pi / 2$ and from equation (2.15), we obtain $e^{i \varphi}=i e^{i \beta}$. Applying these relations, we get

$$
H_{e f}=\frac{1}{2}\left(\begin{array}{cc}
\lambda_{0} & 0 \\
0 & \lambda_{0}
\end{array}\right)+\frac{\Omega}{2}\left(\begin{array}{cc}
0 & -i e^{-i \beta} \\
i e^{i \beta} & 0
\end{array}\right) .
$$

Then, using equation (2.17), we obtain the optimal time evolution as $\tau=|\alpha / \Omega|$, and from equation (2.19), we get $\Omega=|\Omega| e^{i \arg \alpha}$. This example has a simple geometric interpretation. As can be easily shown, the final state $\boldsymbol{n}_{f}$ is obtained from the initial state $\boldsymbol{n}_{i}$ by rotating the complex Bloch sphere $S_{c}^{2}$ through the complex angle $\alpha$ around the axes defined by the vector $\boldsymbol{n}$. It should be noted that for $\Im \alpha=\Im \beta=0$, we have $\Im \Omega=0$, and the Hamiltonian (2.21) coincides with the optimal Hamiltonian, yielding the shortest time evolution $\tau_{m}$ for the unitary evolution. As we will show in the following sections, $\tau_{m}$ gives the upper bound of the time evolution for the generic non-Hermitian Hamiltonian.

The other interesting example is when the initial and final states are orthogonal to each other $(\alpha=\pi)$. The smallest time $\tau_{p}$ required to evolve from a given initial state $\left|\psi_{i}\right\rangle$ to the orthogonal final state $\left|\psi_{f}\right\rangle$ is called the passage time [3,4]. Inserting $\alpha=\pi$ into equation 2.18 , we obtain

$$
\tau_{p}=\left|\frac{i}{\Omega} \ln \frac{Z-\Omega}{Z+\Omega}\right|
$$

Let us consider some limiting cases, starting with the saddle point $Z=0$ (see Fig. 1). Computation yields $\tau_{p}=\pi /|\Omega|$. This can be interpreted as the Hermitian limit of the nonHermitian system. As can be seen in Fig. 1, the evolution of the non-Hermitian quantum system is faster than that of the corresponding Hermitian system, satisfying the same eigenvalue constraint. Moreover, the passage time $\tau_{p} \rightarrow 0$ while $|Z| \rightarrow \infty$.

Next, we find that $\tau_{p} \rightarrow \infty$ at the points $Z= \pm \Omega$ (see Fig. 1). To understand this result, we note that $Z=\Omega$ implies $\theta=0$ and that $Z=\Omega$ yields $\theta=\pi$. For both cases, $\theta=0$ and $\theta=\pi$, the wave function (2.13) becomes

$$
|\psi(t)\rangle=e^{-i\left(\lambda_{0} \pm \Omega\right) t / 2}\left|\psi_{i}\right\rangle
$$

Thus, only phase and amplitude of the wave function are changed; otherwise, the system in the same initial state remains intact. This explains the divergence of the passage time at the points $Z= \pm \Omega$. This is easy to understand by looking at the example of the half-spin particle in the uniform magnetic field, when the spin is eqnarrayed with the magnetic field. 

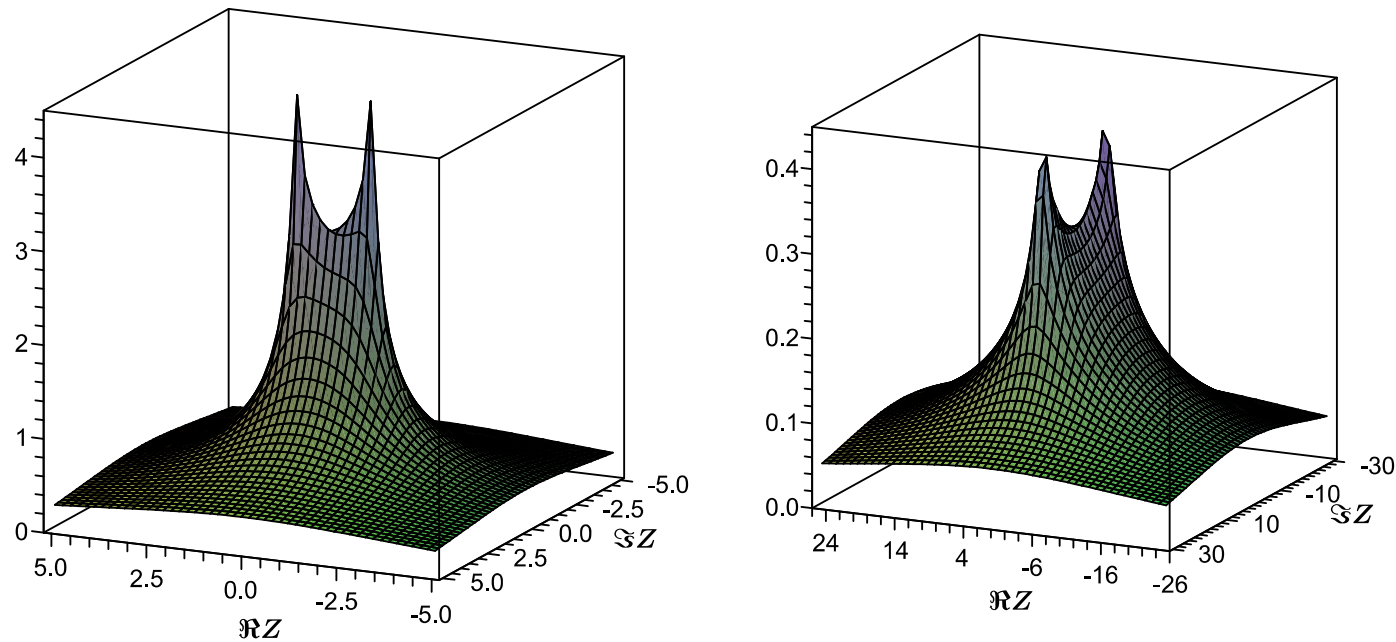

Figure 1. Plot of the passage time $\tau_{p}$ versus $\Re Z$ and $\Im Z$. Left panel: $\Re \Omega=1, \Im \Omega=0.1$. Right panel: $\Re \Omega=1, \Im \Omega=10$. As can be seen from the plots, the singularity occurs at the points $Z= \pm \Omega$ and $\tau_{p}=\pi /|\Omega|$ at the saddle point $Z=0$.

\section{Quantum evolution in the vicinity of the exceptional point}

In the presence of exceptional points characterized by coalescence of eigenvalues and corresponding eigenvectors, non-Hermitian physics differs essentially from Hermitian physics [20, 25, 26]. Since for the Hermitian operators, the coalescence of eigenvalues results in different eigenvectors, exceptional points do not exist for Hermitian systems. The related degeneracies, referred to as 'conical intersections', are known also as 'diabolic points'. In the context of Berry phase, the diabolic point is associated with the 'fictitious magnetic monopole' located at the diabolic point $[27,28]$. In turn, the exceptional points are associated with the 'fictitious complex magnetic monopoles' [29].

For a two-dimensional system (2.4), the eigenvalues coalesce when $\Omega=0$. Writing the complex vector $\boldsymbol{\Omega}$ as $\boldsymbol{\Omega}=\boldsymbol{r}-i \boldsymbol{\delta}$, where $\boldsymbol{r}$ and $\boldsymbol{\delta}$ are real, one can recast the effective Hamiltonian of equation (2.4) in the form

$$
H_{e f}=\frac{\lambda_{0}}{2} \mathbf{1}+\frac{1}{2} \boldsymbol{r} \cdot \boldsymbol{\sigma}-\frac{i}{2} \boldsymbol{\delta} \cdot \boldsymbol{\sigma} .
$$

As can be seen, the degeneracy points are defined by the following equation:

$$
r^{2}-\delta^{2}-2 i r \delta \cos \gamma=0
$$

where $\gamma$ denotes the angle between $\boldsymbol{\delta}$ and $\boldsymbol{r}=(x, y, z)$. Furthermore, it is convenient to use cylindrical coordinates $(\rho, z, \varphi)$, in which $\Omega=\sqrt{\rho^{2}+z^{2}-\delta^{2}-2 i z \delta}$, and equation (3.2) reads: $\rho^{2}+z^{2}-\delta^{2}-2 i z \delta=0$. There are two solutions to this equation. The first one is $\rho=z=\delta=0$, and the corresponding degeneracy point located at the origin of coordinates is the diabolic point. The second solution, given by $z=0$ and $\rho^{2}-\delta^{2}=0$, defines the exceptional point. Generally speaking, the exceptional "point", being realized as a cone in the four-parameter space $(x, y, z, \delta)$, is not a point in the convenient sense, and all possible one(zero)-dimensional cases can be obtained by the conic sections. For instance, for a fixed $\delta$, we obtain the exceptional point as a circle of radius $|\delta|$, lying in the plane $z=0$. It should be noted that in the recent literature, the term "exceptional point" is applied not only to the particular case when the exceptional "point" is indeed a point but to the general case when the non-Hermitian degeneracy is realized as a submanifold in the parameter space [30, 31, 32, 33]. In the following, we will stick to this more general interpretation of the exceptional point. 
As follows from equations (2.9)-(2.13), at the exceptional point, the quantum evolution is described by

$$
|\psi(t)\rangle=\left(1-\frac{\delta t}{2\left(1-\cos \frac{\alpha}{2}\right)}\right) e^{-i \lambda_{0} t}\left|\psi_{i}\right\rangle+\frac{\delta t}{2\left(1-\cos \frac{\alpha}{2}\right)} e^{-i \lambda_{0} t}\left|\psi_{f}\right\rangle .
$$

Without loss of generality, we further assume $\delta>0$. The computation of the time evolution at the exceptional point then yields

$$
\tau=\frac{2}{\delta}\left|\left(1-\cos \frac{\alpha}{2}\right)\right|
$$

In what follows, imposing the eigenvalue constraint as $|\Omega|=$ const, we restrict ourselves by consideration of the orthogonal initial $\left|\psi_{i}\right\rangle$ and final states $\left|\psi_{f}\right\rangle$. Substituting $\alpha=\pi$ into equation (2.13) and taking into account equations (2.15), (2.16), we obtain

$$
|\psi(t)\rangle=\left(\cos \frac{\Omega t}{2}-i \cos \theta \sin \frac{\Omega t}{2}\right) e^{-i \lambda_{0} t}\left|\psi_{i}\right\rangle+\sin \theta \sin \frac{\Omega t}{2} e^{-i \lambda_{0} t}\left|\psi_{f}\right\rangle .
$$

Now recalling that $\cos \theta=(z-i \delta) / \Omega$ and setting $z=0$, we observe that in the vicinity of the exceptional point, there are two different regimes, depending on the relation between $\rho$ and $\delta$.

For $\rho>\delta$, we have $\Omega=\sqrt{\rho^{2}-\delta^{2}}>0$, and inserting $\cos \theta=(z-i \delta) / \Omega$ into equation (3.3), we obtain

$$
|\psi(t)\rangle=\left(\cos \frac{\Omega_{0} t}{2}-\frac{\delta}{\Omega_{0}} \sin \frac{\Omega_{0} t}{2}\right) e^{-i \lambda_{0} t}\left|\psi_{i}\right\rangle+\frac{\rho}{\Omega_{0}} \sin \frac{\Omega_{0} t}{2} e^{-i \lambda_{0} t}\left|\psi_{f}\right\rangle,
$$

where $\Omega_{0}=\left|\rho^{2}-\delta^{2}\right|^{1 / 2}$ denotes the Rabi frequency. If, in addition, $\Im \lambda_{0}=0$, then the eigenvalues of the non-Hermitian Hamiltonian (3.1) are real, and we obtain the $\mathcal{P} \mathcal{T}$-symmetric Hamiltonian widely discussed in the recent literature in connection with the quantum brachistochrone problem $[2,5,7,8,9,10,11,12,34,35]$. Then, applying (2.18), we get (see Fig. 2)

$$
\tau_{p}=\frac{2}{\Omega_{0}} \arctan \left(\frac{\Omega_{0}}{\delta}\right)
$$

As can be shown, the passage time $\tau_{p}$ is bounded above and below as follows: $2 / \sqrt{\Omega_{0}^{2}+\delta^{2}}<$ $\tau_{p}<\min \left\{\pi / \Omega_{0}, 2 / \delta\right\}$. This improves the estimation of the passage time obtained in [2].

As follows from equation (3.4), for $\Omega_{0} \gg \delta$, the passage time has the same behavior as for the equivalent Hermitian system: $\tau_{p} \sim \pi / \Omega_{0}$, and if $\Omega_{0} \ll \delta$, one obtains $\tau_{p} \sim 2 / \delta$. At the exceptional point $\Omega_{0}=0$ (and $\left.\rho=\delta\right)$ :

$$
|\psi(t)\rangle=\left(1-\frac{\delta t}{2}\right) e^{-i \lambda_{0} t}\left|\psi_{i}\right\rangle+\frac{\delta t}{2} e^{-i \lambda_{0} t}\left|\psi_{f}\right\rangle .
$$

From here, we get $\tau_{p}=2 / \delta$ and $\tau_{p} \rightarrow 0$ if $\delta \rightarrow \infty$. Thus, in contrast to the Hermitian systems, the passage time may be arbitrarily small; this, of course, implies that $\rho, \delta$ becomes large. This is possible due to the hyperbolic nature of $\Omega_{0}$ considered as function of $\rho$, and $\delta$ and agrees with the results obtained in Bender et al. [2]. As a result, the equivalent Hermitian system in the limit $\Omega_{0} \rightarrow 0$ yields $\tau_{p}=\pi / \Omega_{0} \rightarrow \infty$.

Now assuming $\rho<\delta$, we have $\Omega=i \Omega_{0}$ and

$$
|\psi(t)\rangle=\left(\cosh \frac{\Omega_{0} t}{2}-\frac{\delta}{\Omega_{0}} \sinh \frac{\Omega_{0} t}{2}\right) e^{-i \lambda_{0} t}\left|\psi_{i}\right\rangle+\frac{\rho}{\Omega_{0}} \sinh \frac{\Omega_{0} t}{2} e^{-i \lambda_{0} t}\left|\psi_{f}\right\rangle .
$$



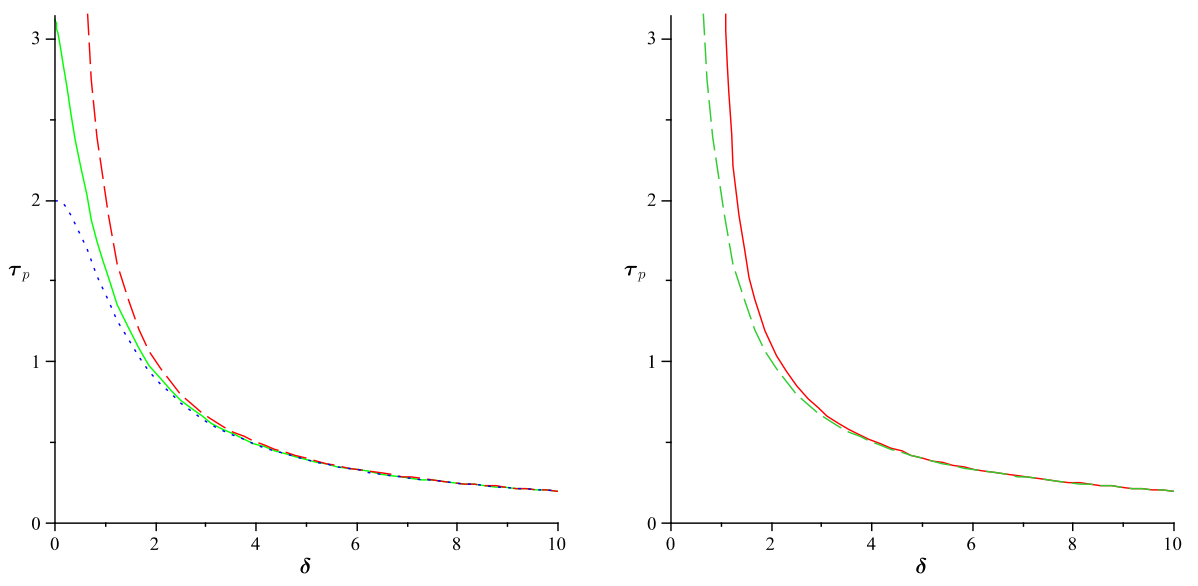

Figure 2. Plot of passage time $\tau_{p}$ vs. $x=\delta\left(\Omega_{0}=1\right)$. Left panel: $\tau_{p}=\left(2 / \Omega_{0}\right) \arctan \left(\Omega_{0} / \delta\right)$ ( $\operatorname{solid}$ green line), $\tau=2 / \sqrt{\Omega_{0}^{2}+\delta^{2}}$ (dotted blue line) and $\tau=2 / \delta$ (dashed red line). Right panel: $\tau_{p}=$ $\left(2 / \Omega_{0}\right) \tanh ^{-1}\left(\Omega_{0} / \delta\right)$ (solid red line) and $\tau=2 / \delta$ (dashed green line). It is seen that in both cases, $\tau_{p}$ is asymptotically approximated by $\tau=2 / \delta$.

Computation of the passage time yields

$$
\tau_{p}=\frac{2}{\Omega_{0}} \tanh ^{-1}\left(\frac{\Omega_{0}}{\delta}\right) .
$$

As seen in Fig. 2, the passage time is bounded below as follows: $\tau_{p}>2 / \delta$, and it follows from equation (3.5) that $\tau_{p} \sim 2 / \delta$ in the limit $\Omega_{0} \ll \delta$. At the exceptional point, we obtain the same result as above: $\tau_{p}=2 / \delta$ and

$$
|\psi(t)\rangle=\left(1-\frac{\delta t}{2}\right) e^{-i \lambda_{0} t}\left|\psi_{i}\right\rangle+\frac{\delta t}{2} e^{-i \lambda_{0} t}\left|\psi_{f}\right\rangle
$$

As an illustrative example, we consider a two-level dissipative system driven by a periodic electromagnetic field $\mathbf{E}(t)=\Re(\mathcal{E}(t) \exp (i \nu t))$. In the rotating wave approximation, after removing the explicit time dependence of the Hamiltonian and the average effect of the decay terms, the Schrödinger equation reads [36, 37]

$$
i\left(\begin{array}{c}
\dot{u}_{1} \\
\dot{u}_{2}
\end{array}\right)=\frac{1}{2}\left(\begin{array}{cc}
-i \lambda+\Delta-i \delta & 2 V^{*} \\
2 V & -i \lambda-\Delta+i \delta
\end{array}\right)\left(\begin{array}{l}
u_{1} \\
u_{2}
\end{array}\right),
$$

where $\lambda=\left(\gamma_{a}+\gamma_{b}\right) / 2$, with $\gamma_{a}, \gamma_{b}$ being decay rates for upper and lower levels, respectively, $\Delta=E_{a}-E_{b}-\nu, \delta=\left(\gamma_{a}-\gamma_{b}\right) / 2, V=\boldsymbol{\mu} \cdot \mathcal{E}$, and $\boldsymbol{\mu}$ is the dipole matrix element.

The choice $\mathcal{E}(t)=\mathcal{E}_{0} \exp (i \omega t)$ yields $V(t)=V_{0} \exp (i \omega t)$, where $V_{0}=\boldsymbol{\mu} \cdot \mathcal{E}_{0}$, and we assume further that $V_{0}>0$. The solution of equation (3.6) with this choice of $\mathcal{E}$ can be written as

$$
|u(t)\rangle=C_{1}(t) e^{-i(\omega-i \lambda) t / 2}\left|u_{\uparrow}\right\rangle+C_{2}(t) e^{i(\omega+i \lambda) t / 2}\left|u_{\downarrow}\right\rangle,
$$

where $\left|u_{\uparrow}\right\rangle=\left(\begin{array}{l}1 \\ 0\end{array}\right)$ and $\left|u_{\downarrow}\right\rangle=\left(\begin{array}{l}0 \\ 1\end{array}\right)$, denote the up/down states, respectively,

$$
|C(t)\rangle=\left(\begin{array}{l}
C_{1}(t) \\
C_{2}(t)
\end{array}\right)=\left(\begin{array}{cc}
\cos \frac{\Omega t}{2}-i \cos \theta \sin \frac{\Omega t}{2} & -i \sin \theta \sin \frac{\Omega t}{2} \\
-i \sin \theta \sin \frac{\Omega t}{2} & \cos \frac{\Omega t}{2}+i \cos \theta \sin \frac{\Omega t}{2}
\end{array}\right)\left(\begin{array}{l}
C_{1}(0) \\
C_{2}(0)
\end{array}\right)
$$

and we set $\cos \theta=(\Delta-\omega-i \delta) / \Omega, \Omega=\left(\rho^{2}+(\Delta-\omega-i \delta)^{2}\right)^{1 / 2}, \rho=2 V_{0}$. 
As can be shown, the state $|C(t)\rangle$ satisfies the Schrödinger equation

$$
i \frac{\partial|C\rangle}{\partial t}=H_{r}|C\rangle
$$

written in a co-rotating reference frame, where the Hamiltonian $H_{r}$ takes the form

$$
H_{r}=\frac{\Omega}{2}\left(\begin{array}{cc}
\cos \theta & \sin \theta \\
\sin \theta & -\cos \theta
\end{array}\right) .
$$

Let $|u(t)\rangle$ be the solution of equation (3.6) with the initial state at $t=0$ taken as $\left|u_{\uparrow}\right\rangle$ and the final state of the system at a later time $t$ being $\left|u_{\uparrow}\right\rangle$ or $\left|u_{\downarrow}\right\rangle$. Then following Baker [38], we compute the probability $P_{\uparrow \uparrow}\left(P_{\downarrow \uparrow}\right)$ that the system is in the state $\left|u_{\uparrow}\right\rangle\left(\left|u_{\downarrow}\right\rangle\right)$, respectively:

$$
P_{\uparrow \uparrow}=\left|\cos \frac{\Omega t}{2}-i \cos \theta \sin \frac{\Omega t}{2}\right|^{2} e^{-\lambda t}, \quad P_{\downarrow \uparrow}=\left|\sin \theta \sin \frac{\Omega t}{2}\right|^{2} e^{-\lambda t} .
$$

For the resonance frequencies, $\omega=\Delta$, we have $\Omega=\left(\rho^{2}-\delta^{2}\right)^{1 / 2}$, and $\cos \theta=-i \delta / \Omega_{0}$. There are two different regimes, depending on the relation between $\rho$ and $\delta$. For $\rho>\delta$, we have a coherent tunneling process

$$
P_{\uparrow \uparrow}=e^{-\lambda t}\left(\cos \frac{\Omega_{0} t}{2}-\frac{\delta}{\Omega_{0}} \sin \frac{\Omega_{0} t}{2}\right)^{2}, \quad P_{\downarrow \uparrow}=e^{-\lambda t} \frac{\rho^{2}}{\Omega_{0}^{2}} \sin ^{2} \frac{\Omega_{0} t}{2},
$$

where $\Omega_{0}=\left|\rho^{2}-\delta^{2}\right|^{1 / 2}$ denotes the Rabi frequency. On the other hand, for $\rho<\delta$, there is incoherent tunneling

$$
P_{\uparrow \uparrow}=e^{-\lambda t}\left(\cosh \frac{\Omega_{0} t}{2}-\frac{\delta}{\Omega_{0}} \sinh \frac{\Omega_{0} t}{2}\right)^{2}, \quad P_{\downarrow \uparrow}=e^{-\lambda t} \frac{\rho^{2}}{\Omega_{0}^{2}} \sinh ^{2} \frac{\Omega_{0} t}{2} .
$$

At the exceptional point, $\Omega_{0}=0$, and both regimes yield

$$
P_{\uparrow \uparrow}=\left(1-\frac{\delta t}{2}\right)^{2} e^{-\lambda t}, \quad P_{\downarrow \uparrow}=\left(\frac{\delta t}{2}\right)^{2} e^{-\lambda t} .
$$

This is in accordance with the results obtained by Stafford and Barrett and Dietz et al. [39, 40]. Moreover, the decay behavior predicted by equations (3.7)-(3.8) has been observed in the experiment with a dissipative microwave billiard [40].

We find that for $\rho>\delta$, the passage time required to transform the state $\left|u_{\uparrow}\right\rangle$ into the state $\left|u_{\downarrow}\right\rangle$ is given by $\tau_{p}=\left(2 / \omega_{0}\right) \arctan \left(\omega_{0} / \delta\right)$. Similarly, for $\rho<\delta$, we obtain $\tau_{p}=\left(2 / \omega_{0}\right) \tanh ^{-1}\left(\omega_{0} / \delta\right)$. At the exceptional point, for both regimes, the passage time is found to be $\tau_{p}=2 / \delta$ (see Fig. 2 ).

\section{Fubini-Study metric on the complex Bloch sphere and the brachistochrone problem}

In this section, we will consider the quantum non-Hermitian brachistochrone problem in the context of the geometric approach developed by Anandan and Aharonov [41]. Let $|\psi(t)\rangle$ and $\langle\tilde{\psi}(t)|$ satisfy the Schrödinger equation and its adjoint equation, respectively:

$$
i \frac{\partial}{\partial t}|\psi(t)\rangle=H|\psi(t)\rangle, \quad-i \frac{\partial}{\partial t}\langle\tilde{\psi}(t)|=\langle\tilde{\psi}(t)| H,
$$


where $H$ is a non-Hermitian Hamiltonian, and the standard normalization is held, $\langle\tilde{\psi}(t) \mid \psi(t)\rangle=1$. We define the complex energy variance as

$$
\Delta E^{2}=\left\langle\tilde{\psi}\left|H^{2}\right| \psi\right\rangle-(\langle\tilde{\psi}|H| \psi\rangle)^{2} .
$$

Now, applying the Taylor expansion to $|\psi(t+d t)\rangle$ and using equation (4) and its time derivative, we obtain

$$
\langle\tilde{\psi}(t) \mid \psi(t+d t)\rangle^{2}=1-\Delta E^{2} d t^{2}+\mathcal{O}\left(d t^{3}\right) .
$$

Then, introducing the complex metric as $d s^{2}=4\left(1-\langle\tilde{\psi}(t) \mid \psi(t+d t)\rangle^{2}\right)$, we obtain

$$
d s^{2}=4 \Delta E^{2} d t^{2}=4 d s_{\mathrm{FS}}^{2},
$$

where

$$
d s_{\mathrm{FS}}^{2}=\langle d \tilde{\psi}|(1-P)| d \psi\rangle
$$

is a natural generalization of the Fubini-Study line element to the non-Hermitian quantum mechanics, $P=|\psi\rangle\langle\tilde{\psi}|$ being the projection operator to the state $|\psi\rangle$. It is easy to show that the complex-valued metric (4.2) is gauge invariant, i.e., it does not depend on the particular choice of the complex phase defined by the map: $|\psi\rangle \rightarrow e^{i \alpha}|\psi\rangle,\langle\tilde{\psi}| \rightarrow e^{-i \alpha}\langle\tilde{\psi}|, \alpha \in \mathbb{C}$. We define the distance between two given states $\left|\psi_{0}\right\rangle$ and $\left|\psi_{1}\right\rangle$ as

$$
s=2 \int_{\mathcal{C}}|\Delta E(t)| d t
$$

where the integration is performed along a given curve $\mathcal{C}$ connecting $\left|\psi_{0}\right\rangle$ and $\left|\psi_{1}\right\rangle$.

In the two-dimensional case, the complex-valued Fubini-Study element has a nice geometric interpretation. We define a complex distance between two nearby Bloch vectors $\boldsymbol{n}(x)$ and $\boldsymbol{n}(x+d x)$ by

$$
\Delta^{2}(x, x+d x)=1-\boldsymbol{n}(x) \cdot \boldsymbol{n}(x+d x) .
$$

Then, applying Taylor expansion,

$$
\boldsymbol{n}(x+d x)=\boldsymbol{n}(x)+\partial_{i} \boldsymbol{n}(x) d x^{i}+\frac{1}{2} \partial_{i} \partial_{j} \boldsymbol{n}(x) d x^{i} d x^{j}+\cdots,
$$

and using $\boldsymbol{n} \cdot \boldsymbol{n}=1$, we obtain, up to second-order terms,

$$
\boldsymbol{n}(x+d x)=1-d \boldsymbol{n}(x) \cdot d \boldsymbol{n}(x) .
$$

This yields

$$
d s^{2}=d \boldsymbol{n} \cdot d \boldsymbol{n}=g_{i j} d x^{i} d x^{j},
$$

where $g_{i j}=\partial_{i} \boldsymbol{n} \cdot \partial_{j} \boldsymbol{n}$, and the length of any curve $\mathcal{C}$ on $S_{c}^{2}$ is given by

$$
L(\mathcal{C})=\int_{\mathcal{C}}|\sqrt{d \boldsymbol{n} \cdot d \boldsymbol{n}}|
$$

Denoting $\boldsymbol{n}=(\sin \zeta \cos \nu, \sin \zeta \sin \nu, \cos \zeta)$, where $\zeta, \nu \in \mathbb{C}$, we recast (4.4) as

$$
d s^{2}=d \zeta^{2}+\sin ^{2} \zeta d \nu^{2} .
$$


Finally, using the definition of the complex Bloch vector $\boldsymbol{n}=\langle\tilde{\psi}|\boldsymbol{\sigma}| \psi\rangle$, we find that the metric on the complex Bloch sphere $S_{c}^{2}$ can be written as

$$
d s^{2}=d \boldsymbol{n} \cdot d \boldsymbol{n}=4 d s_{\mathrm{FS}}^{2}=4\langle d \tilde{\psi}|(1-P)| d \psi\rangle,
$$

where $d s_{\mathrm{FS}}^{2}=\langle d \tilde{\psi}|(1-P)| d \psi\rangle$ is the Fubini-Study line element.

Strictly speaking, $g_{i j}$ being a complex-valued tensor does not define a proper metric on the complex Bloch sphere. However, the advantage of this definition is that, contrary to the Kähler metric, the complex "metric" (4.4) is invariant under the gauge transformation $|\psi\rangle \rightarrow e^{i \alpha}|\psi\rangle$, $\langle\tilde{\psi}| \rightarrow e^{-i \alpha}\langle\tilde{\psi}|$, where $\alpha \in \mathbb{C}$.

For the Hamiltonian (2.20), the straightforward computation yields

$$
2 \Delta E=\Omega \sin \theta
$$

and, using equations (2.18) and (4.3), we obtain

$$
L=2|\Delta E| \tau=\left|2 \sin \theta \arctan \left(\frac{i \sin ^{2} \frac{\alpha}{2}}{\cos \frac{\alpha}{2} \sqrt{\cos ^{2} \theta-\sin ^{2} \frac{\alpha}{2}}-\cos \theta}\right)\right| .
$$

To compare our results with the $\mathcal{P} T$-symmetric model widely discussed in the literature (see, e.g., $[6,7,8,9,10,11,12,13,14])$, we choose $\theta=\pi / 2+i \eta$ and assume $\Im \lambda_{0}=\Im \varphi=0$ to write the effective Hamiltonian of equation (2.20) as

$$
H_{\text {eff }}=\frac{1}{2}\left(\begin{array}{cc}
\lambda_{0} & 0 \\
0 & \lambda_{0}
\end{array}\right)+\frac{\Omega}{2}\left(\begin{array}{cc}
-i \sinh \eta & e^{-i \varphi} \cosh \eta \\
e^{i \varphi} \cosh \eta & i \sinh \eta
\end{array}\right) .
$$

Next, we set $\lambda_{0}=r \cos \gamma, \Omega \sinh \eta=r \sin \gamma$, and $\Omega \cosh \eta=\omega$. Denoting $\delta=r \sin \gamma$, we obtain the Hamiltonian of the system in its conventional form (see, e.g., $[2,5]$ ):

$$
H_{\mathrm{eff}}=\frac{1}{2}\left(\begin{array}{cc}
r e^{-i \gamma} & \omega e^{-i \varphi} \\
\omega e^{i \varphi} & r e^{i \gamma}
\end{array}\right)
$$

where $\Omega=\sqrt{\omega^{2}-r^{2} \sin ^{2} \gamma}=\sqrt{\omega^{2}-\delta^{2}}$. In this particular case, the complex Bloch sphere becomes the one-sheeted two-dimensional hyperboloid $\mathbb{H}^{2}$ with the indefinite metric given by [24]

$$
d s^{2}=\cosh ^{2} \rho d \nu^{2}-d \rho^{2}
$$

where $-\infty<\rho<\infty$ and $0 \leq \nu<2 \pi$ are the inner parameters on $\mathbb{H}^{2}$. It should be noted that the interval (4.9) can be obtained from equation (4.5) by the substitution $\zeta \rightarrow \pi / 2+i \rho$, and we assume $\Im \nu=0$.

The amount of time $\tau$ required to evolve the initial state $\left|\psi_{i}\right\rangle$ into the final state $\left|\psi_{f}\right\rangle$ can be found from equation (2.18) by substituting $\theta=\pi / 2+i \eta$. The computation yields

$$
\tau=\frac{2}{\Omega} \arctan \left(\frac{\sin ^{2} \frac{\alpha}{2}}{\cos \frac{\alpha}{2} \sqrt{\sinh ^{2} \eta+\sin ^{2} \frac{\alpha}{2}}+\sinh \eta}\right),
$$

and from equation (4.7), we obtain

$$
L=2 \cosh \eta \arctan \left(\frac{\sin ^{2} \frac{\alpha}{2}}{\cos \frac{\alpha}{2} \sqrt{\sinh ^{2} \eta+\sin ^{2} \frac{\alpha}{2}}+\sinh \eta}\right) .
$$


To study the spin-flip, we choose the initial state as $\left|\psi_{i}\right\rangle=\left|u_{\uparrow}\right\rangle$. Then, substituting $\alpha=\pi$ into equation (4.10), we find the time interval

$$
\tau_{\downarrow}=\frac{2}{\Omega} \arctan \frac{1}{\sinh \eta}=\frac{2}{\Omega} \arctan \frac{\Omega}{\delta}, \quad \tau_{\uparrow}=\frac{2 \pi}{\Omega}-\frac{2}{\Omega} \arctan \frac{\Omega}{\delta},
$$

necessary for the first spin flip from up to down and back. For all values $\Omega \in[0, \infty)$, we have $2 / \delta \leq \tau_{\downarrow} \leq \pi / \Omega$. Thus, the passage time $\tau_{p}=\tau_{\downarrow}$ lies below the Anandan-Aharonov lower bound, $\tau_{\downarrow} \leq \pi / \Omega$ for a spin-flip evolution in a Hermitian system [42], and $\tau_{p}$ reaches its minimum value $\tau_{\min }=2 / \delta$ at the exceptional point. In addition, the total time for a spin-flip evolution, $|\uparrow\rangle \rightarrow|\downarrow\rangle \rightarrow|\uparrow\rangle$, remains invariant: $\tau=\tau_{\downarrow}+\tau_{\uparrow}=2 \pi / \Omega$. This is in accordance with the results of previous studies $[34,10]$.

Introducing the variable $\kappa=\arctan (\delta / \Omega)=\arctan (\sinh \eta)$, we reproduce our results in the more familiar form, widely known in the literature (see, e.g., $[2,3,4,10]$ ):

$$
\tau_{\downarrow}=\frac{\pi-2 \kappa}{\Omega}, \quad \tau_{\uparrow}=\frac{\pi+2 \kappa}{\Omega} .
$$

We see that in the Hermitian limit, $\eta \rightarrow 0(\delta \ll \Omega)$ that implies $\kappa \rightarrow 0$, the passage time is given by $\tau_{p}=\pi / \Omega$. In the other limiting case $\eta \rightarrow \infty(\Omega \ll \delta)$, the angle $\kappa$ approaches $\pi / 2$, and $\tau_{p}$ tends to zero. In terms of variable $\kappa$, the relation $\Omega \cosh \eta=\omega$ becomes $\Omega=\omega \cos \kappa$. Furthermore, if the energy constraint $E_{+}-E_{-}=\Omega$ is held fixed, in order to have the passage to the limit $\kappa \rightarrow \pi / 2$, one must require $\omega \gg \Omega$. It then follows from the relation $\Omega^{2}=\omega^{2}-\delta^{2}=$ const that one must require $\delta \gg \Omega$.

Similar consideration of the distance between the initial and the final states yields

$$
L_{p}=2 \cosh \eta \arctan \left(\frac{1}{\sinh \eta}\right)=\frac{\pi-2 \kappa}{\cos \kappa} .
$$

In the limit $\kappa \rightarrow \pi / 2$, we get $L_{p} \rightarrow 2$, and in the Hermitian limit, $\kappa \rightarrow 0$, we have $L_{p} \rightarrow \pi$. It then follows from equation (4.11), that the distance between $\left|u_{\uparrow}\right\rangle$ and $\left|u_{\downarrow}\right\rangle$, being measured on the one-sheeted two-dimensional hyperboloid $\mathbb{H}^{2}$ with the indefinite metric of equation (4.9), is bounded as follows: $2 \leq L_{p} \leq \pi$.

Inserting $\theta=\pi / 2+i \eta$ into (4.6), we obtain $2 \Delta E=\Omega \cos \eta$. Then substituting this result into equation (4.1), we find that for the non-Hermitian Hamiltonian (4.8), the evolution speed $v=d s / d t$ is given by $v=\omega=\Omega \cosh \eta$. Hence, $v \geq v_{g}$, where $v_{g}=\Omega$ is the speed of the geodesic evolution [24]. Similar consideration of the quantum-mechanical system governed by the Hermitian Hamiltonian yields $v=\Omega \sin \theta$, and, obviously, $v \leq v_{g}$. This proves that nonHermitian quantum mechanics can be faster than Hermitian quantum mechanics. Moreover, since for any complex angle $\theta$, there exists $\theta_{0}$ determined by the equation $\cos \Re \theta_{0}=\sinh \Im \theta_{0}$ such that $v=|\Omega \sin \theta| \geq|\Omega|=v_{g}$ if $|\Im \theta| \geq\left|\Im \theta_{0}\right|$, this conclusion is applied to an arbitrary non-Hermitian Hamiltonian (see Fig. 3).

Our results are in agreement with those obtained previously by Bender et al. [2]. However, interpreting the obtained results for $\tau$ requires care. Indeed, as was pointed out by Mostafazadeh [7], to compare the time evolution for the non-Hermitian and Hermitian Hamiltonians and to conclude in which case the evolution is faster, one should not only impose the same set of constraints for both cases but also fix the geodesic distance between initial and final states.

In what follows, we consider in detail a spin-flip for the Hamiltonian (4.8) with two types of constraints: a) the energy variance $\Delta E^{2}=\left\langle\tilde{\psi}\left|H^{2}\right| \psi\right\rangle-(\langle\tilde{\psi}|H| \psi\rangle)^{2}$ held fixed [1], and b) the difference between the largest and smallest energy eigenvalues has a fixed value, $E_{+}-E_{-}=$ const [2]. In terms of the parameters $(\Omega, \omega)$, the energy constraints are written as follows: 


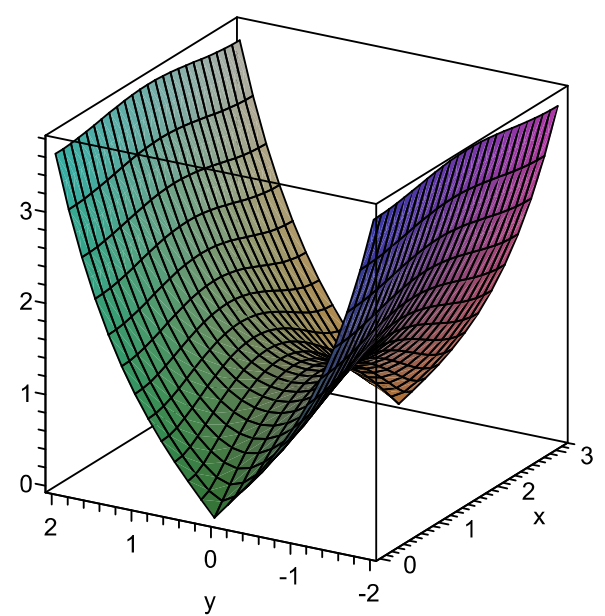

Figure 3. Plot of the evolution speed $|v| /|\Omega|$ versus $x=\Re \theta$ and $y=\Im \theta$. As can be observed, for any $\theta: 0 \leq \Re \theta \leq \pi$, there exists the angle $\theta_{0}$ such that $|v| /|\Omega| \geq 1$, if $|\Im \theta| \geq\left|\Im \theta_{0}\right|$.

$2 \Delta E=\omega$ and $E_{+}-E_{-}=\Omega$. Then, using the relation $\cosh \eta=\omega / \Omega$, we find that the passage time can be recast as

$$
\tau_{p}=\frac{2}{\Omega} \arctan \frac{\Omega}{\sqrt{\omega^{2}-\Omega^{2}}} .
$$

As can be observed, under the constraint $\omega=$ const, the time evolution is bounded by $2 / \omega \leq$ $\tau_{p} \leq \pi / \omega$. Here, the passage time reaches its minimum value $\tau_{\min }=2 / \omega$ at the exceptional point $\Omega=0(\eta \rightarrow \infty)$. The maximum, $\tau_{\max }=\pi / \omega$, is obtained for $\Omega=\omega$. Since $\Omega=\omega$ implies $\eta=0$, this case corresponds to the Hermitian Hamiltonian. Next, we let the energy constraint be $E_{+}-E_{-}=\Omega=$ const. Then, as follows from equation (4.12), $\tau_{p} \leq \pi / \Omega$, and, just as above, the passage time achieves the maximum $\tau_{\max }=\pi / \Omega$ at the point $\omega=\Omega$ corresponding to the Hermitian Hamiltonian. For $\omega \gg \Omega$, we obtain $\tau_{p} \approx 2 / \omega$, and $\tau_{p}$ vanishes in the limit $\omega \rightarrow \infty$.

Similar consideration of the distance between the initial and final states yields

$$
L_{p}=\omega \tau=\frac{2 \omega}{\Omega} \arctan \frac{\Omega}{\sqrt{\omega^{2}-\Omega^{2}}}
$$

and, under the constraint $\omega=$ const, we have $2 \leq L \leq \pi$. The upper bound $L_{\max }=\pi$, being identical to the geodesic distance between the initial and final states defined either on the Bloch sphere $S^{2}$ or on the one-sheeted hyperboloid $\mathbb{H}^{2}$ [24], is thus achieved for the Hermitian Hamiltonian $(\Omega=\omega)$. The lower bound, $L_{\min }=2$, is obtained at the exceptional point $(\Omega=0)$. This agrees with our general conclusions regarding the behavior of the system in the vicinity of the exceptional point (see Section 3). Next, imposing the constraint $\Omega=$ const, we obtain the same result $2 \leq L_{p} \leq \pi$. The minimum of $L_{p}$ corresponds to the limit $\omega \gg \Omega$, and, just as above, the upper bound is achieved for the Hermitian Hamiltonian $(\omega=\Omega)$.

We now turn our discussion to the recent controversy around the possibility of achieving faster evolution in a quantum-mechanical system governed by a non-Hermitian $\mathcal{P} \mathcal{T}$-symmetric Hamiltonian as compared to the equivalent Hermitian system [2, 6, 7, 9]. It should be noted that the critique of the results obtained in [2] is essentially based on the following theorem [7]: The lower bound on the travel time (upper bound on the speed) of unitary evolutions is a universal quantity, independent of whether the evolution is generated by a Hermitian or a non-Hermitian Hamiltonian. Analyzing the proof of this theorem, one can see that it is founded on the following assumption: the minimal travel time is realized by quantum evolution along the geodesic path in the Hilbert space joining initial and final states. 
This is true in the case of the Hermitian Hamiltonian; however, for a non-Hermitian Hamiltonian, the situation is quite different [24]. Let $\mathbf{n}_{i}$ and $\mathbf{n}_{f}$ denote antipodal states on the Bloch sphere $S^{2}$, and $\mathbf{m}_{i}$ and $\mathbf{m}_{f}$ denote corresponding (antipodal) states on the one-sheeted hyperboloid $\mathbb{H}^{2}$. Then, the geodesic distance $L_{g}$ between $\mathbf{n}_{i}$ and $\mathbf{n}_{f}$ calculated over the Bloch sphere $S^{2}$ is identical to the geodesic distance between $\mathbf{m}_{i}$ and $\mathbf{m}_{f}$ computed over the one-sheeted hyperboloid $\mathbb{H}^{2}$ (for detailed calculations, see [24]). Moreover, under the same set of constraints, the amount of time $\tau_{g}$ required to evolve $\mathbf{n}_{i}$ into $\mathbf{n}_{f}$ on the Bloch sphere is equal to that required to evolve $\mathbf{m}_{i}$ into $\mathbf{m}_{f}$ on $\mathbb{H}^{2}$ by geodesic evolution. This is in accordance with the conclusions made by Mostafazadeh in [7]. However, as we have shown above, in the case of the Hermitian Hamiltonian, $\tau_{g}$ is the lower bound on the time evolution, and, for the non-Hermitian Hamiltonian, it yields only the upper bound on the time evolution. Thus, in non-Hermitian quantum mechanics, the evolution of a system is indeed faster than in Hermitian quantum mechanics, subject to the same energy constraint.

\section{Conclusion}

In this paper, we considered the non-Hermitian quantum brachistochrone problem for the generic non-Hermitian Hamiltonian and focused our attention on the geometric aspects of the problem. We demonstrated that for the generic non-Hermitian Hamiltonian, the quantum brachistochrone problem can be effectively formulated on the complex Bloch sphere $S_{c}^{2}$, enabling the latter to be considered as the quantum phase space of the related two-level system. In particular, for the effective non-Hermitian Hamiltonian with a real energy spectrum, the corresponding quantum phase space is represented by the one-sheeted hyperboloid $\mathbb{H}^{2}$.

As noted in $[4,5]$, in the Hermitian quantum brachistochrone problem, the lower bound on the time evolution interval $\tau$, being proportional to the geodesic distance between the initial and final states on the Bloch sphere, is determined using the Fubini-Studi metric on $\mathbb{C} P^{1} \cong S^{2}$. We have shown that the geodesic distance $L_{g}$ between antipodal states $\mathbf{n}_{i}$ and $\mathbf{n}_{f}$ calculated over the Bloch sphere $S^{2}$ is identical to the geodesic distance between corresponding antipodal states $\mathbf{m}_{i}$ and $\mathbf{m}_{f}$ calculated over the one-sheeted hyperboloid $\mathbb{H}^{2}$. Moreover, the amount of time $\tau_{g}$ required to evolve $\mathbf{n}_{i}$ into $\mathbf{n}_{f}$ on the Bloch sphere is equal to that required to evolve $\mathbf{m}_{i}$ into $\mathbf{m}_{f}$ on $\mathbb{H}^{2}$ by the geodesic evolution. This is in accordance with the conclusions made in [7]. However, in the case of the Hermitian Hamiltonian, $\tau_{g}$ is the lower bound on the time evolution, and, for the non-Hermitian Hamiltonian, it yields only the upper bound on the time evolution. Furthermore, while for a quantum-mechanical system governed by the Hermitian Hamiltonian the evolution, speed $v$ is bounded by $v \leq v_{g}$, where $v_{g}$ is the speed of the geodesic evolution, for a non-Hermitian quantum system with the same energy constraint, we have $v \geq v_{g}$. This proves that in non-Hermitian quantum mechanics, evolution can be faster than in Hermitian quantum mechanics [2].

\section{Acknowledgements}

The author is grateful to C. Bender, D. Brody, U. Günther, A. Mostafazadeh, I. Rotter and B.F. Samsonov for helpful discussions and comments. This work is supported by research grants SEP-PROMEP 103.5/04/1911. 


\section{References}

[1] Carlini A., Hosoya A., Koike T., Okudaira Y., Time-optimal quantum evolution, Phys. Rev. Lett. 96 (2006), 060503, 4 pages.

[2] Bender C.M., Brody D.C., Jones H.F., Meister B.K., Faster than Hermitian quantum mechanics, Phys. Rev. Lett. 98 (2007), 040403, 4 pages, quant-ph/0609032.

[3] Brody D.C., Elementary derivation for passage times, J. Phys. A: Math. Gen. 36 (2003), 5587-5593, quant-ph/0302067.

[4] Brody D.C., Hook D.W., On optimum Hamiltonians for state transformations, J. Phys. A: Math. Gen. 39 (2006), L167-L170, quant-ph/0601109.

[5] Bender C.M., Brody D.C., Optimal time evolution for Hermitian and non-Hermitian Hamiltonians, in Time in Quantum Mechanics II, Editor J.G. Muga, Lecture Notes in Phys., Springer, to appear, arXiv:0808.1823.

[6] Assis P.E.G., Fring A., The quantum brachistochrone problem for non-Hermitian Hamiltonians, J. Phys. A: Math. Theor. 41 (2008), 244002, 12 pages, quant-ph/0703254.

[7] Mostafazadeh A., Quantum brachistochrone problem and the geometry of the state space in pseudoHermitian quantum mechanics, Phys. Rev. Lett. 99 (2007), 130502, 4 pages, arXiv:0706.3844.

[8] Mostafazadeh A., Hamiltonians generating optimal-speed evolutions, Phys. Rev. A 79 (2009), 014101, 4 pages, arXiv:0804.4755.

[9] Bender C.M., Brody D.C., Jones H.F., Meister B.K., Comment on the quantum brachistochrone problem, arXiv:0804.3487.

[10] Günther U., Samsonov B.F., The $\mathcal{P} \mathcal{T}$-symmetric brachistochrone problem, Lorentz boosts and non-unitary operator equivalence classes, Phys. Rev. A 78 (2008), 042115, 9 pages, arXiv:0709.0483.

[11] Günther U., Samsonov B.F., Naimark-dilated $\mathcal{P} \mathcal{T}$-symmetric brachistochrone, Phys. Rev. Lett. 101 (2008), 230404, 4 pages, arXiv:0807.3643.

[12] Günther U., Rotter I., Samsonov B.F., Projective Hilbert space structures at exceptional points, J. Phys. A: Math. Theor. 40 (2007), 8815-8833, arXiv:0704.1291.

[13] Martin D., Is $\mathcal{P} \mathcal{T}$-symmetric quantum mechanics just quantum mechanics in a non-orthogonal basis?, quant-ph/0701223.

[14] Rotter I., The brachistochrone problem in open quantum systems, J. Phys. A: Math. Theor. 40 (2007), 14515-14526, arXiv:0708.3891.

[15] Weisskopf V.F., Wigner E.P., Berechnung der natürlichen Linienbreite auf Grund der Diracschen Lichttheorie, Z. f. Physik 63 (1930), 54-73.

[16] Weisskopf V.F., Wigner E.P., Über die natürliche Linienbreite in der Strahlung des harmonischen Oszillators. Z. f. Physics 65 (1930), 18-29.

[17] Aharonov Y., Massar S., Popescu S., Tollaksen J., Vaidman L., Adiabatic measurements on metastable systems, Phys. Rev. Lett. 77 (1996), 983-987, quant-ph/9602011.

[18] Plenio M.B., Knight P.L., The quantum-jump approach to dissipative dynamics in quantum optics, Rev. Modern Phys. 70 (1998), 101-144, quant-ph/9702007.

[19] Carollo A., Fuentes-Guridi I., França Santos M., Vedral V., Geometric phase in open system, Phys. Rev. Lett. 90 (2003), 160402, 4 pages, quant-ph/0301037.

[20] Berry M.V., Physics of nonhermitian degeneracies, Czechoslovak J. Phys. 54 (2004), 1039-1047.

[21] Morse P.M., Feshbach H., Methods of theoretical physics, McGraw-Hill, New York, 1953.

[22] Chu S.-I., Wu Z.-C., Layton E., Density matrix formulation of complex geometric quantum phases in dissipative systems, Chem. Phys. Lett. 157 (1989), 151-158.

[23] Chu S.-I., Telnov D.A., Beyond the Floquet theorem: generalized Floquet formalisms and quasienergy methods for atomic and molecular multiphoton processes in intense laser fields, Phys. Rep. 390 (2004), $1-131$.

[24] Nesterov A.I., An optimum Hamiltonian for non-Hermitian quantum evolution and the complex Bloch sphere, arXiv:0806.4646.

[25] Heiss W.D., Exceptional points of non-Hermitian operators, J. Phys. A: Math. Gen. 37 (2004), 2455-2464, quant-ph/0304152. 
[26] Heiss W.D., Exceptional points - their universal occurrence and their physical significance, Czechoslovak J. Phys. 54 (2004), 1091-1099.

[27] Berry M.V., Quantal phase factor accompanying adiabatic changes, Proc. Roy. Soc. London Ser. A 392 (1984), 45-57.

[28] Berry M.V., Dennis M.R., The optical singularities of birefringent dichroic chiral crystals, Proc. Roy. Soc. London Ser. A 459 (2003), 1261-1292.

[29] Nesterov A.I., Aceves de la Cruz F., Complex magnetic monopoles, geometric phases and quantum evolution in vicinity of diabolic and exceptional points, J. Phys. A: Math. Theor. 41 (2008), 485304, 25 pages, arXiv:0806.3720.

[30] Kirillov O.N., Mailybaev A.A., Seyranian A.P., Unfolding of eigenvalue surfaces near a diabolic point due to a complex perturbation, J. Phys. A: Math. Gen. 38 (2005), 5531-5546, math-ph/0411006.

[31] Mailybaev A.A., Kirillov O.N., Seyranian A.P., Geometric phase around exceptional points, Phys. Rev. A 72 (2005), 014104, 4 pages, quant-ph/0501040.

[32] Seyranian A.P., Kirillov O.N., Mailybaev A.A., Coupling of eigenvalues of complex matrices at diabolic and exceptional points, J. Phys. A: Math. Gen. 38 (2005), 1723-1740, math-ph/0411024.

[33] Mailybaev A.A., Kirillov O.N., Seyranian A.P., The Berry phase in a neighborhood of degenerate states, Dokl. Akad. Nauk 406 (2006), 464-468.

[34] Giri P.R., Lower bound of minimal time evolution in quantum mechanics, Internat. J. Theoret. Phys. 47 (2008), 2095-2100, arXiv:0706.3653.

[35] Geyer H.B., Heiss W.D., Scholtz F.G., Non-Hermitian Hamiltonians, metric, other observables and physical implications, arXiv:0710.5593.

[36] Garrison J.C., Wright E.M., Complex geometrical phases for dissipative systems, Phys. Lett. A 128 (1988), 177-181.

[37] Lamb W.E., Schlicher R.R., Scully M.O., Matter-field interaction in atomic physics and quantum optics, Phys. Rev. A 36 (1987), 2763-2772.

[38] Baker H.C., Non-Hermitian quantum theory of multiphoton ionization, Phys. Rev. A 30 (1984), 773-793.

[39] Stafford C.A., Barrett B.R., Simple model for decay of superdeformed nuclei, Phys. Rev. C 60 (1999), 051305, 4 pages, nucl-th/9906052.

[40] Dietz B., Friedrich T., Metz J., Miski-Oglu M., Richter A., Schäfer F., Stafford C.A., Rabi oscillations at exceptional points in microwave billiards, Phys. Rev. E 75 (2007), 027201, 4 pages, cond-mat/0612547.

[41] Anandan J., Aharonov Y., Geometry of quantum evolution, Phys. Rev. Lett. 65 (1990), 1697-1700.

[42] Aharonov Y., Anandan J., Phase change during a cyclic quantum evolution, Phys. Rev. Lett. 58 (1987), 1593-1596. 